\title{
Über optische Konstanten und elektrischen Widerstand dicker Metallschichten ${ }^{1}$
}

\author{
Von Karl WeIsS \\ Aus dem Institut für theoretische Physik der Universität Köln \\ (Z. Naturforschg. 3 a, 143-147 [1948]; eingegangen am 28. Juli 1947)
}

\begin{abstract}
In der vorliegenden Arbeit wurde versucht, möglichst absolute Werte für die optischen Konstanten von Gold, Silber und Kupfer zu erreichen. Dabei wurden die Spiegel mit besonderer Sorgfalt hergestellt, wodurch reproduzierbare Werte im Haupteinfallswinkel und -azimut erzielt wurden. Wie aus den mitgeteilten Tabellen ersichtlich ist, konnten dadurch die durchschnittlichen Fehler im Haupteinfallswinkel auf maximal 3' und im Hauptazimut auf maximal 5' beschränkt werden. Die von $\mathrm{K}$ r e tzm a n n benutzte optische Meßapparatur wurde weiter vervollkommnet. Es wurde keine Abhängigkeit der optischen Konstanten von den Aufdampfzeiten und Spiegelunterlagen festgestellt. So können die vom Verf. erhaltenen Zahlen für Gold und Silber als Absolutwerte angesehen werden. Die Widerstandsmessungen stimmten bei Silber bis zu einer gewissen Schichtdicke mit denen des massiven Metalls nahezu überein.
\end{abstract}

$\mathrm{B}$ ei älteren und neueren Messungen der optischen Konstanten dicker Metallschichten weichen die Werte sehr stark voneinander $a b^{\mathbf{1 - 1 8}}$. Dies läßt sich teils dadurch erklären, daß die einzelnen Beobachter ihre Spiegel auf verschiedene Art und Weise herstellten und so mehr oder weniger reine MetalIschichten bekamen ${ }^{2-11}$. In neueren Arbeiten werden die Spiegel nur noch durch Verdampfen des zu untersuchenden Metalls erhalten ${ }^{12-18}$. In einzelnen Arbeiten wurde dabei eine Abhängigkeit der optischen Konstanten von den Aufdampfbedingungen beobachtet ${ }^{13}$. 'Es schien deshalb die Frage berechtigt, ob diese Streuungen durch Änderungen des strukturellen Aufbaus bedingt sind oder auf Verunreinigungen zurückgeführt werden können. In der vorliegenden Arbeit wurde versucht, diese Frage zu klären. Es wurden hierzu die Metalle Gold, Silber und Kupfer noch einmal in dem Spektralbereich 500-1000 m optisch untersucht.

Da ein Zusammenhang zwischen optischen Kon-

1 Auszug aus der Diss. Köln 1939/40, die infolge äußerer Umstände nicht früher veröffentlicht werden konnte.

2 R. S. M i n o r, Ann. Physik (4) 10, 581 [1903].

3 P. D r ude, Wiedemanns Ann. Physik 39, 481 [1890].

4 P. D r ude, Wiedemanns Ann. Physik 64, 161 [1898].

5 W. M e i e r, Ann. Physik 31, 1017 [1910].

6 G. P f e s tor f, Ann. Physik 81, 906 [1926].

7 L. K. O p p itz, Physic. Rev. (2) 10, 156 [1917].

8 A. O. T o o l, Physic. Rev. 31, 1 [1910].

- A. Försterling u. Fréederi cksz, Ann. Physik 40, 201 [1913]. stanten und spezifischem Widerstand besteht und frühere Messungen des letzteren bedeutend höhere Werte lieferten als diejenigen, die man für das massive Metall erhielt, wurde der spezifische Widerstand von Silber gleichzeitig gemessen.

Ursprünglich wurde die von $\mathrm{Kr}$ etz m an $\mathrm{n}^{\mathbf{1 3}}$ beschriebene Aufdampfapparatur benutzt, bei der sich eine beträchtliche Abhängigkeit der optischen Eigenschaften von den Aufdampfbedingungen zeigte. Zur Überprüfung des Vakuums wurde während des Aufdampfens der Druck gemessen. Es konnte beobachtet werden, daß bei schlechtem Vakuum erhaltene Schichten kleinere Werte für die gemessenen Haupteinfallswinkel $\bar{\varphi}$ und Hauptazimute $\bar{\psi}$, aus denen sich die optischen Konstanten errechnen, ergaben. Eine solche merkliche Vakuumverschlechterung ging parallel der Aufdampfzeit. Auf Grund dieser Beobachtungen lag die Vermutung nahe, daß die Änderungen in $\bar{\varphi}$ und $\bar{\psi}$ durch Verunreinigungen bedingt sind (namentlich durch Fett), die durch die zum Verdampfen angewandte Heizung flüchtig werden und sich dann auf den Spiegeln niederschlagen.

Es wurde daher eine neue Hochvakuum-Aufdampfanlage gebaut. In dieser erschien ein Aufdampfraum

10 L. R. Ingersoll, Astrophysic. J. 32, 265 [1910].

11 E. Hàg en u. H. R u bens, Ann. Physik (4) 8, 16 [1902]; (4) 11, 873 [1903].

12 G. H a s s, Ann. Physik (5) 31, 245 [1938]; Optik 1, 8 [1946].

${ }_{13}$ R. K r etz m a n n, Ann. Physik 37, 303 [1940].

14 F. O d e n b a c h, Diss. Köln 1939.

15 H. M. O'B r y o n, J. opt. Soc. America 26, 122 [1936].

16 B. O'Brien, Physic. Rev. (2) 27, 93 [1926].

17 L. S. O r n s te in u. I. H. v. d. Ve en, Physica 3, 289 [1936].

18 J. K r a u t k r äme r, Ann. Physik (5) 32, 543 [1938]. 
ohne jegliche Fettdichtung erforderlich. Dazu wurde in die äußere Glasglocke ein unten geschliffener Glaszylinder von $20 \mathrm{~cm}$ Durchmesser und $28 \mathrm{~cm}$ Höhe ohne Fett auf die gläserne Grundplatte gesetzt. Nach oben wurde der Zylinder mit einem etwa $8 \mathrm{~cm}$ hohen und runden Glasgefäß abgedeckt. Der innere Glaszylinder sowie der äußere Restraum wurden mittels zweier Hg-Diffusionspumpen luftleer gepumpt. Das Verdampfen der Metalle erfolgte aus drei Wolfram- bzw. Molybdänblechen, die zwischen zwei stromführenden Eisenbalken eingeklemmt waren. Die Stromzuführungen wurden von innen durch Wasser gekühlt. Zum Schutz gegen die Wärmestrahlen waren die Kittstellen im inneren Glaszylinder mit Kupferblech und der

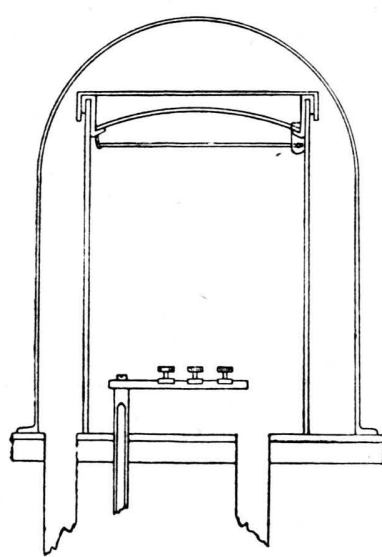

Abb. 1. Schematische Darstellung der Aufdampfapparatur. gesamte Boden mit für Wärmestrahlen undurchlässigem Filterglas abgedeckt. Die Spiegel lagen auf einem nahezu als Kreisbogen ausgebildeten Gestell. Eine unmittelbar unter den Spiegeln angebrachte Glasplatte schützte dieselben vor Bestäuben beim Einschmelzen und Verdampfen des Metalls. Vor dem Aufdampfen wurde die Abdeckplatte mit einem Elektromagneten nach unten geklappt. Glimmer konnte nicht als Abschirmmaterial gebraucht werden, da er bei der im Innern herrschenden

Temperatur schon calcinierte und gaste. Beim Verdampfen wurde darauf geachtet, daß die Schiffchen nicht restlos leer dampften. Der Abstand der Spiegel von dem verdampfenden Metall betrug $23 \mathrm{~cm}$. Die Messung des Druckes erfolgte im Innenraum mit einem Ionisationsmanometer. Nach 1 Stde. Pumpzeit wurde ein Druck von 2.10-5 Torr erreicht, der beim Aufdampfen der Schichten nahezu konstant blieb.

Als optische Meßeinrichtung wurde eine ähnliche Apparatur wie die von $\mathrm{Kretzmann^{13 }}$ beschriebene verwendet. Besondere Sorgfalt wurde auf die Herstellung von monochromatischem Licht gelegt. Da ein einfacher, von Kretzmann gebrauchter Monochromator wegen Streulichtes nicht den Anforderungen entsprach, wurde ein $\mathrm{Z}$ e i $ß$ scher Zweiprismenapparat benutzt, der zur Erzielung größerer Lichtstärken umgebaut worden war. Die geringen Abweichungen der Ergebnisse dieser Arbeit von den Resultaten von Kretzmann sind vermutlich z. Tl. durch den Unterschied in der Monochromasie bedingt. Versuche, die mit einer Vormonochromatisierung gemacht wurden, ergaben keine nennenswerte Verbesserung. So betrug im ganzen Spektralbereich die Differenz im Haupteinfallswinkel bei derselben Spiegelgarnitur mit und ohne Vormonochromator maximal 2', während im Hauptazimut vollkommene
Übereinstimmung herrschte. Aus Intensitätsgründen wurde dann bei der endgültigen Messung auf die Vormonochromatisierung verzichtet. Die Messung erfolgte mit einem Vierspiegelapparat, bei dem die Strahlrichtung erhalten blieb. Als Analysator wurde ein erstklassiges Glan-Thompson-Prisma von $20 \mathrm{~mm}^{2}$ Querschnitt verwendet. Die Justierung der Vierspiegelapparatur erfolgte so, daß eine Grobjustierung mit der Wasserwaage vorausging. Dann wurden die Arm- und Tischwinkel justiert und die Spiegel zum Bezugsspiegel 4 senkrecht gestellt. Jetzt erst wurden Analysator und Polarisator angebracht und die Schwingungsrichtungen parallel und senkrecht zur Apparateachse sehr sorgfältig ermittelt. Versuche, am Polarisationsspektrometer durch verschiedene Polarisationsprismen die Hauptschwingungsrichtungen $\mathrm{zu}$ bestimmen, ergaben Abweichungen bis

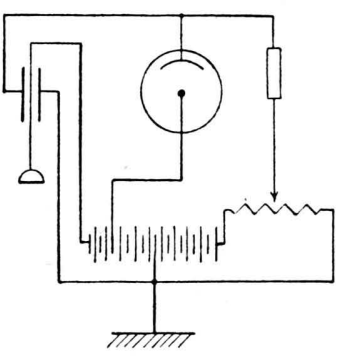

Abb. 2. Elektrometer- und Photozellenschaltung.

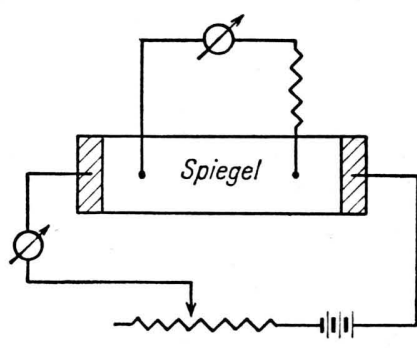

Abb. 3. WiderstandsMeßanordnung.
14'. Daraufhin wurde die Nullstellung mit dem Brewsterschen Winkel und einer Kalkspatplatte, deren optische Achse senkrecht zum Strahlengang lag, festgestellt. Nach beiden Methoden stimmten die Hauptschwingungsrichtungen bis auf $1^{\prime}$ überein. Ein Glan-Thompson-Prisma, das denselben Wert für die Nullstellung ergab, wurde dann später zur Justierung der Vierspiegelapparatur verwendet.

Die Lichtintensitäten wurden mit einer AEGVakuum-Photozelle in Verbindung mit einem Elektrometer in der üblichen Weise gemessen (Abb. 2).

Anfänglich wurde der Widerstand mit einer technischen Meßbrücke bestimmt. Es zeigte sich, daß bei Silber der Kontaktwiderstand zwischen Schicht und Zuführungsklemmen in derselben Größenordnung lag wie der des zu untersuchenden Spiegels (s. Tab. 3). Deswegen wurde der spezifische Widerstand mit einer neuen Apparatur ermittelt, die im Prinzip auf einer Strom- und Spannungsmessung beruhte und in ähnlicher Ausführung schon von $\mathrm{R}$ i ed e benutzt wurde (Abb.3). Die zur Bestimmung des Widerstandes benutzten Spiegel hatten eine Länge von $8 \mathrm{~cm}$ und eine Breite von 1,2 cm. Die Spannungsmessung erfolgte durch Abgriff der Potentialdifferenz zwischen zwei in Längsrichtung liegenden Punkten. Bei der Widerstandsmessung war es äußerst wichtig, daß die Spiegel homogen waren. Dieses wurde auch tatsächlich durch den großen Abstand der Spiegel von den Glühblechen und die kreisförmige Anordnung 


\begin{tabular}{|c|c|c|c|c|c|c|c|c|c|c|c|c|}
\hline \multirow[b]{2}{*}{$\begin{array}{c}\text { Wellen- } \\
\text { länge } \\
\text { m } \mu\end{array}$} & \multicolumn{4}{|c|}{$\mathrm{S}$ il b e r } & \multicolumn{4}{|c|}{$\mathrm{G} \circ \mathrm{ld}$} & \multicolumn{4}{|c|}{$\mathrm{K} \mathrm{upfer}$} \\
\hline & $\begin{array}{l}\text { Mittel- } \\
\text { werte } \\
\text { für } \bar{\varphi}\end{array}$ & $\begin{array}{l}\text { durch- } \\
\text { schn. } \\
\text { Fehler }\end{array}$ & $\begin{array}{l}\text { Mittel- } \\
\text { werte } \\
\text { für } \bar{\psi}\end{array}$ & $\begin{array}{l}\text { durch- } \\
\text { schn. } \\
\text { Eehler }\end{array}$ & $\begin{array}{l}\text { Mittel- } \\
\text { werte } \\
\text { für } \bar{\varphi}\end{array}$ & $\begin{array}{l}\text { durch- } \\
\text { schn. } \\
\text { Eehler }\end{array}$ & $\begin{array}{l}\text { Mittel- } \\
\text { werte } \\
\text { für } \bar{v}\end{array}$ & $\begin{array}{l}\text { durch- } \\
\text { schn. } \\
\text { Fehler }\end{array}$ & $\begin{array}{l}\text { Mittel- } \\
\text { werte } \\
\text { für } \bar{\varphi}\end{array}$ & $\begin{array}{l}\text { durch- } \\
\text { schn. } \\
\text { Fehler }\end{array}$ & $\begin{array}{l}\text { Mittel- } \\
\text { werte } \\
\text { für } \bar{\psi}\end{array}$ & $\begin{array}{l}\text { durch- } \\
\text { schn. } \\
\text { Fehler }\end{array}$ \\
\hline 500 & $73^{0} \quad 9^{\circ}$ & $4^{\prime}$ & $44^{\circ} 23^{\prime}$ & $3^{\prime}$ & & & & & & & & \\
\hline 550 & 7450 & 3 & 4428 & 2 & $69^{\circ} 36^{\prime}$ & $5^{\prime}$ & $41^{\circ} 30^{\prime}$ & $8^{\prime}$ & $70^{0} 50^{\prime}$ & $1^{\prime}$ & $37^{\circ} 26^{\prime}$ & $2^{\prime}$ \\
\hline 600 & 7616 & 4 & 4430 & 2 & 7239 & 1 & 4313 & 7 & $73 \quad 2$ & 2 & 4323 & 4 \\
\hline 650 & $\begin{array}{ll}77 & 23\end{array}$ & 3 & 4429 & 2 & 7439 & 1 & 4353 & 3 & 7520 & 3 & 4356 & 4 \\
\hline 700 & 7821 & 3 & 4427 & 2 & $\begin{array}{ll}76 & 14\end{array}$ & 1 & $44 \quad 5$ & 5 & 7651 & 2 & 4400 & 4 \\
\hline 750 & $79 \quad 8$ & 3 & 4426 & 2 & 7724 & 1 & 44 & 5 & 7754 & 2 & $44 \quad 2$ & 4 \\
\hline 800 & 7950 & 3 & 4426 & 2 & 7820 & 2 & 44 & 4 & 7847 & 3 & 44 & 5 \\
\hline 850 & 8025 & 2 & 4425 & 2 & $79 \quad 5$ & 1 & 44 & 4 & 7931 & 2 & 44 & 5 . \\
\hline 900 & 8055 & 2 & 4425 & 2 & 7942 & 2 & $44 \quad 8$ & 4 & $80 \quad 6$ & 1 & 43 & 6 \\
\hline 950 & 8123 & 2 & 4426 & 2 & 8019 & 0 & $\begin{array}{ll}44 & 9\end{array}$ & 3 & 8037 & 3 & 44 & 6 \\
\hline 1000 & 8149 & 2 & 4428 & 2 & 8049 & 1 & $44 \quad 10$ & 4 & 818 & 4 & $\begin{array}{ll}44 & 7\end{array}$ & 6 \\
\hline
\end{tabular}

Tab. 1. Aus den Einzelmessungen errechnete Mittelwerte für den Haupteinfallswinkel $\bar{\varphi}$ und das Hauptazimut $\bar{\psi}$.

\begin{tabular}{|c|c|c|c|c|c|c|c|c|c|c|c|c|}
\hline \multirow{2}{*}{$\begin{array}{l}\text { Wellen- } \\
\text { länge } \\
\text { mu }\end{array}$} & \multicolumn{4}{|c|}{$\mathrm{S}$ i I b e r } & \multicolumn{4}{|c|}{$\mathrm{G} \circ \mathrm{ld}$} & \multicolumn{4}{|c|}{$\mathrm{Kupfer}$} \\
\hline & $n$ & $n k$ & $k$ & $\begin{array}{c}R \% \\
\text { berechnet }\end{array}$ & $n$ & $n k$ & $k$ & $\begin{array}{c}R \% \\
\text { berechnet }\end{array}$ & $n$ & $n k$ & $k$ & $\begin{array}{c}R \% \\
\text { berechnet }\end{array}$ \\
\hline 500 & 0,071 & 0,215 & 3,020 & 97,3 & & & & & & & & \\
\hline 550 & 0,069 & 0,240 & 3,429 & 97,9 & 0,331 & 0,768 & 2,324 & 81,6 & 0,756 & 1,861 & 2,462 & 66,7 \\
\hline 600 & 0,072 & 0,276 & 3,348 & 98,2 & 0,200 & $0, \check{5} 80$ & 2,897 & 91,9 & 0,186 & 0,553 & 2,980 & 92,5 \\
\hline 650 & 0,080 & 0,343 & 4,257 & 98,4 & 0,142 & 0,481 & 3,374 & 95,5 & 0,142 & 0,508 & 3,570 & 95,9 \\
\hline 700 & 0,093 & 0,433 & 4,645 & 98,4 & 0,131 & 0,503 & 3,842 & 96,7 & 0,150 & 0,606 & 4,049 & 96,6 \\
\hline 750 & 0,103 & 0,518 & 5,005 & 98,4 & 0,140 & 0,598 & 4,266 & $y 7,1$ & 0,157 & 0,701 & 4,463 & 96,9 \\
\hline 800 & 0,110 & 0,596 & 5,409 & 98,6 & 0,149 & 0,693 & 4,654 & 97,4 & 0,170 & 0,825 & 4,840 & 97,2 \\
\hline 850 & 0,121 & 0,694 & 5,757 & 98,6 & 0,157 & 0,784 & 4,993 & 97,6 & 0,182 & 0,952 & 5,222 & 97,3 \\
\hline 900 & 0,128 & 0,777 & 6,089 & 98,7 & 0,166 & 0,887 & 5,335 & 97,8 & 0,190 & 1,056 & 5,569 & 97,7 \\
\hline 950 & 0,130 & 0,842 & 6,476 & 98,8 & 0,174 & 0,990 & 5,691 & 97,9 & 0,197 & 1,165 & 5,900 & 97,8 \\
\hline 1000 & 0,129 & 0,882 & 6,829 & 98,9 & 0,179 & 1,084 & 6,044 & 98,1 & 0,197 & 1,236 & 6,272 & 98,0 \\
\hline
\end{tabular}

Tab. 2. Die optischen Konstanten von Silber, Gold und Kupfer.

erreicht. Als Kriterium konnte die Konstanz des Spannungsabfalls beim Verschieben angesehen werden. Die Spiegel wurden nach dem Erkalten sofort gemessen.

Die Berechnung der optischen Konstanten erfolgte durch Bestimmung des Haupteinfallswinkels $\bar{\varphi}$ und des. Hauptazimuts $\bar{\psi}$. Es handelte sich dabei um eine Intensitätsmessung. Nach dreifacher Reflexion gilt

$$
\frac{R_{s}^{\prime \prime \prime}}{R_{p}^{\prime \prime \prime}}=Q^{3} e^{-i 3 \delta} \cdot \frac{E_{s}}{E_{p}} ;
$$

dabei ist $R_{s}^{\prime \prime} / R_{p}^{\prime \prime}$ das Verhältnis der Komponenten senkrecht und parallel zur Einfallsebene nach dreimaliger Reflexion, $E_{s} / E_{p}$ das Verhältnis der Komponenten senkrecht und parallel zur Einfallsebene beim einfallenden Strahl, $\delta$ die Phasendifferenz und $\varrho=\operatorname{tg} \bar{\psi}$.
Für linear polarisiertes Licht, dessen Polarisationsebene um $45^{\circ}$ gegen die Einfallsebene geneigt ist, wird unter dem Haupteinfallswinkel

$$
\begin{gathered}
\frac{E_{s}}{E_{p}}=1, \quad 3 \delta=\frac{3}{2} \pi, \\
\frac{R_{s}^{\prime \prime \prime}}{R_{p}^{\prime \prime \prime}}=-\varrho^{3} i
\end{gathered}
$$

wobei das austretende Licht elliptisch polarisiert ist und die Hauptachsen der Ellipse parallel und senkrecht zur Einfallsebene stehen. Steht der Analysator $45^{\circ}$ und $135^{\circ}$ zur Einfallsebene geneigt, so muß die Photozelle die gleichen Stromwerte liefern, da die Lichtintensitäten gleich sind.

Die Bestimmung des Hauptazimuts $\bar{\psi}$ erfolgt unter dem Haupteinfallswinkel $\bar{\varphi}$ so, daß man $E_{s} / E_{y}^{\prime}=\operatorname{tg} \alpha$ setzt und $\alpha$ so lange ändert, bis das 
austretende Licht zirkular polarisiert ist. Es ist dann

$$
\begin{gathered}
\frac{R_{s}^{\prime \prime \prime}}{R_{p}^{\prime \prime \prime}}=-i \varrho^{3} \operatorname{tg} a, \quad \frac{R_{s}^{\prime \prime \prime}}{R_{p}^{\prime \prime \prime}}=1, \\
\operatorname{tg} a=\frac{1}{Q^{3}}=\frac{1}{\operatorname{tg}^{3} \psi} .
\end{gathered}
$$

Aus $\alpha$ wird dann $\bar{\psi}$ berechnet.

Die Berechnung der optischen Konstanten erfolgte nach den strengen D r u d e schen Formeln ${ }^{4}$.

$$
\begin{gathered}
n^{2}=\frac{\sin ^{2} \bar{\varphi} \operatorname{tg}^{2} \bar{\varphi} \sin 4 \bar{\psi}}{2 \operatorname{tg} \% / 2}, \\
\operatorname{tg} \%=\frac{\sin ^{2} \bar{\varphi} \operatorname{tg}^{2} \bar{\varphi} \sin 4 \bar{\psi}}{\sin ^{2} \bar{\varphi} \operatorname{tg}^{2} \bar{\varphi} \cos 4 \bar{\psi}+\sin ^{2} \bar{\psi}}, \\
n k=n^{2} \varkappa=\frac{\sin ^{2} \bar{\varphi} \operatorname{tg}^{2} \bar{\varphi} \sin 4 \bar{\psi}}{2}, \\
\varkappa=\operatorname{tg} \frac{\psi}{2}, \\
R=\frac{(n-1)^{2}+(\varkappa n)^{2}}{(n+1)^{2}+(* n)^{2}} .
\end{gathered}
$$

Die Ergebnisse der Messungen an Silber, Gold und Kupfer zeigen die Tab. 1 und 2.

Bei den drei Metallen Silber, Gold und Kupfer zeigte sich, daß die optischen Konstanten unabhängig von den Aufdampfbedingungen wurden. Entsprechend der Zunahme des Reinheitsgrades konnte eine Zunahme des Haupteinfallswinkels und Hauptazimutes beobachtet werden. Die aufgedampften Metalle waren chemisch reinst. Die Dicke der Schichten lag bei Silber zwischen 120 bis $200 \mathrm{~m} \mu$, bei Gold und Kupfer zwischen 300 bis $400 \mathrm{m \mu}$. Da in der vorliegenden Arbeit ganz besondere Sorgfalt sowohl auf den optischen Teil als auf die Herstellung der Spiegel verwendet wurde, kann man die so erhaltenen optischen Konstanten als Absolutwerte für die Metalle Gold und Silber auffassen. Es ist nicht anzunehmen, daß in der kurzen Meßzeit die Metalle irgendeine Anderung erfahren, die die Konstanten beeinflußt. Die Werte für Kupfer müßten noch einmal im Hochvakuum direkt gemessen werden. Die optischen Konstanten stimmen gut mit denen neuerer Arbeiten überein ${ }^{12,13}$.

Die Meßergebnisse waren weitgehendst reproduzierbar und die Genauigkeit wurde bis jetzt von keinem Beobachter an aufgedampften Schichten erreicht. Der Unterschied gegenüber dem massiven
Metall ist wohl darauf zurückzuführen, daß durch dessen Bearbeitung noch Reste des Polier- und Reinigungsmittels auf ihm vorhanden sind. Andere Beobachter erhielten eine Zunahme der Reflexion bei verschiedenen Metallen in Abhängigkeit von dem Reinheitsgrad der aufgedampften Metalle. Diese Erfahrung fand der Verf. bei den drei vorgenannten Metallen bestätigt durch Berechnung der Reflexion aus seinen Werten für die optischen Konstanten. Man kann so umgekehrt die gefundene Reflexion als einen Beweis für die Richtigkeit der gefundenen Absolutwerte für die optischen Konstanter ansehen.

Eine Abhängigkeit von der Unterlage konnte nicht beobachtet werden. Spiegel auf Quarz und Glas ergaben dieselben Meßwerte.

Um den spezifischen Widerstand aus dem gemessenen $\mathrm{zu}$ errechnen, mußte die Dicke der Schichten bestimmt werden. Diese wurde nach zwei verschiedenen Methoden ermittelt. Nach der Newtonschen Ringmethode ist die Dicke gegeben durch:

$$
d=\frac{a_{k}^{\prime}-a_{k}}{a_{k+1}-a_{k}} \frac{\lambda}{2}-\frac{\lambda}{4 \pi}\left(\Delta_{12}-\Delta_{10}\right),
$$

$a_{k}=$ Radius des $k$-ten Ringes auf Glas,

$a_{k}^{\prime}=$ Radius des $k$-ten Ringes auf Metall,

$J_{10}=$ Phasenbeschleunigung bei der Reflexion an Glas in Luft,

$\dot{\Delta}_{12}=$ Phasenbeschleunigung bei der Reflexion an der Metallschicht in Luft.

Nach Fresnel ist:

$$
\operatorname{tg} \Delta_{12}=-\frac{2 k}{n^{2}+k^{2}-1} .
$$

Diese Methode ist mit einer Ungenauigkeit von mindestens $10 \%$ behaftet. Die Dicke wurde deshalb hauptsächlich durch Wägung der aufgedampften Schichten mit einer Mikrowaage bestimmt. Mit der Waage konnten noch $10^{-6} \mathrm{~g}$ gewogen werden. Aus dem Gewicht $G$, der Fläche $F$ und der Dichte $S$ ließ sich dann die Dicke bestimmen : $D=G / F S$.

Die Widerstandsmessung wurde nur für Silber durchgeführt. Es wurde darauf geachtet, daß die Spiegel durch Strom nicht zu stark belastet wurden.

Bei Gold erfolgten wegen Materialmangels keine Messungen. Die Kupferwerte sollen später im 
Vakuum gemessen und erst dann veröffentlicht werden.

In Tab. 3 sind die spezifischen Widerstände in Abhängigkeit von der Schichtdicke dargestellt:

\begin{tabular}{|c|c|c|}
\hline \multirow{2}{*}{$\begin{array}{c}\text { Dicke } D \\
\text { in } m \varphi\end{array}$} & \multicolumn{2}{|c|}{ spezifischer Widerstand $\varrho \cdot 10^{4}$} \\
\cline { 2 - 3 } & Brückenmethode & Aligriffsmethode \\
\hline 700 & 0,0284 & 0,0172 \\
381 & - & 0,0165 \\
394 & - & 0,0159 \\
359 & - & 0,0148 \\
268 & - & 0,0179 \\
139 & - & 0,0198 \\
110 & - & 0,0203 \\
94 & - & 0,0230 \\
65 & & 0,1217 \\
\hline
\end{tabular}

Tab. 3. Spezifischer Widerstand in Abhängigkeit von der Schichtdicke.
Die Werte des spezifischen Widerstandes für Silber stimmen nahezu mit dem am massiven Metall gemessenen überein. Gerechnet wurde mit der normalen Dichte des Metalls. Sollte eine Dichteänderung gegenüber dem massiven Metall vorliegen, so kann sie nur sehr minimal sein. Eine Zunahme des spezifischen Widerstands mit Abnahme der Schichtdicken dürfte durch einen allmählichen Utbergang in eine andere Modifikation des Metalls begründet sein (Übergang in die amorphe Form).

Hrn. Prof. Försterling möchte ich für die Anregung zur vorliegenden Arbeit und seine Unterstützung meinen Dank aussprechen. Ebenfalls danke ich dem Assistenten des Instituts für theoretische Physik, Hrn. Dr. K r a u k r äm er, sowie dem Mechaniker, Hrn. B r a u e, für den schnellen Zusammenbau der Apparaturen.

\title{
Die Abhängigkeit der Reichweite der Elektronen von ihrer Energie
}

\author{
Von Richard Glocker. \\ Aus dem Röntgeninstitut der Technischen Hochschule Stuttgart \\ (Z. Naturforschg. 3a, 147-151 [1948]; eingegangen am 20. Februar 1948)
}

\begin{abstract}
Angabe einer Näherungsformel, nach Art der Flammersfeld schen Formel, für praktische und wahre Reichweiten im Energiebereich von $15 \mathrm{keV}$ bis nahezu $100 \mathrm{MeV}$. Nachweis, daß der Exponent im Whiddingtonschen Gesetz kleiner als 2 ist. Angabe einer Näherungsformel für die spezifische Ionisation der Luft von 4 bis $200 \mathrm{keV}$. Erklärung-der geringen Unterschiede zwischen wahrer, praktischer und maximaler Reichweite.
\end{abstract}

$V$ on Flammersfeld $\mathrm{d}^{1}$ wurde kürzlich für Elektronen von 0 bis $3 \mathrm{MeV}$ eine einfache Beziehung zwischen maximaler Reichweite $R_{\max }$, gemessen in $\mathrm{g} / \mathrm{cm}^{2}$, und Energie $E$, gemessen in $\mathrm{keV}$, angegeben:

$$
E=1920 \sqrt{R_{\text {max }}^{2}+0,22 R_{\text {max }}} .
$$

Die maximale Reichweite, auf deren Definition noch eingegangen wird, eignet sich besonders für Messungen an den kontinuierlichen $\beta$-Spektren der radioaktiven Isotopen. Für andere Aufgaben der Physik, z. B. für die chemischen und biologischen Wirkungen von Röntgen- und $\boldsymbol{\gamma}$-Strahlen, sind die an Folien ermittelten praktischen Reichweiten von unmittelbarer Bedeutung, so daß die

1 A. F l a m m e r s f e ld, Z. Naturforschg. 2a, 370 [1947].
Frage naheliegt, ob sich auch für diese eine solch umfassende Beziehung aufstellen läßt.

An Folien verschiedener Dicke wird die Zahl der durchtretenden Elektronen, z. B. durch die Aufladung eines Faraday-Käfiges, gemessen $\left(\mathrm{Schonland}{ }^{2}\right)$ und in Abhängigkeit von der Foliendicke aufgetragen. Der Schnitt der Verlängerung des geradlinigen Teiles der Kurve mit der Abszissenachse liefert die praktische Reichweite $R_{\mathrm{p}}$ in Abb.1. Bei den Messungen von $\mathrm{Varde \textrm {r } ^ { 3 }}$ bei höheren Energien wurde eine Ionisationskammer benützt. Außer der Zahl der Elektronen geht dann auch noch die Energie, welche ein Elektron beim Austritt aus der Folie hat, in die Messung ein. Die Kurven von leichten Stoffen zeigen einen

2 B. F.I. S c h o n l a n d, Proc. Roy. Soc. [London] Ser. A 104, 235 [1923]; 108, 187 [1925].

3 R. W. Varder, Philos. Mag. J. Sci. 29, 725 [1915]. 